\title{
Right Post-Insertion Management in Pediatrics
}

\author{
Amanda Ullman and Tricia Kleidon
}

\begin{abstract}
Maintaining function throughout the VADs therapeutic dwell requires the use of effective post-insertion care strategies. Some aspects of post-insertion VAD management can be appropriately generalized from adult literature, and this has already been summarized in Chaps. 10-12. This includes the importance of regular assessment of the entire VAD and patients, including the administration set, for signs of complications and device malfunction. Also, previously summarized in Chaps. 2 and 10 is the use of bundles of care, to ensure the practical application of high-quality evidence to the bedside. These bundles are of great importance during the post-insertion phase, when considering the use of skin antisepsis, dressings, securement, and patency procedures. However, the focus of this chapter is on elements of post-insertion
\end{abstract}

A. Ullman $(\bowtie)$

Alliance for Vascular Access Teaching and Research (AVATAR) Group, Menzies Health Institute Queensland, School of Nursing and Midwifery, Griffith University, Brisbane, QLD, Australia e-mail: a.ullman@griffith.edu.au

T. Kleidon

Alliance for Vascular Access Teaching and Research (AVATAR), Queensland Children's Hospital,

Brisbane, QLD, Australia

e-mail: tricia.kleidon@ health.qld.gov.au care that are specific to the pediatric population and their VAD.

\section{Keywords}

Pediatric VAD management - Pediatric catheter flushing $\cdot$ Pediatric skin considerations $\cdot$ Pediatric complications $\cdot$ Pediatric dressing considerations $\cdot$ Pediatric securement

\subsection{Introduction}

As with vascular access devices (VADs) in other populations, insertion day is only the beginning of the pediatric vascular access journey. Reliability in pediatric VAD is of upmost importance. Infants and children need to be able to receive their vascular access-dependent treatments as prescribed. However, a recent meta-analysis demonstrated that after successful insertion, $25 \%$ of pediatric central venous access devices (CVAD) fail prior to completion of treatment due to complications including bloodstream infection, local site infection, phlebitis, thrombosis, dislodgement, and fracture (Ullman et al. 2015c). Single studies have reported pediatric peripheral VAD failure rates of between 25 and 35\%, most commonly from infiltration, phlebitis, occlusion, and extravasation (Malyon et al. 2014; Rozsa et al. 2015). 


\subsection{Pediatric Skin Health, Antisepsis, Dressing, and Securement}

\subsubsection{Pediatric Skin Health}

The skin is the largest organ in the body. Among other functions, the skin plays an important role in the prevention of infection, providing a barrier to environmental pathogens. Impaired skin integrity is common in pediatrics due to agerelated skin pathologies and the sequelae of pediatric conditions. As described in Chap. 13, premature neonatal skin can be extremely fragile, with less developed epidermal layers. Inflammatory skin conditions, such as eczema and other forms of dermatitis, are common during childhood. Health conditions that commonly present during childhood, such as cystic fibrosis (CF) and acute lymphoblastic leukemia (ALL), involve treatment with medications that result in altered skin conditions and altered wound healing.

Children with these complex health conditions rely on the administration of vital medical treatment via their VAD despite loss of skin integrity. Their underlying impaired skin, coupled with the application and removal of adhesive and antiseptic products, results in skin complica- tions surrounding their VAD. Skin complications that present around pediatric VAD include irritant and allergic contact dermatitis, skin tears and blistering, pressure injuries, maceration, and local site infections (Broadhurst et al. 2017) (see Fig. 16.1).

The promotion of pediatric skin health surrounding VAD plays an important role in maintaining VAD function. This can be achieved through the systematic monitoring, prevention, and treatment of VAD-associated skin complications. VAD assessment must include contemporaneous documentation of skin health progression within the patients' medical records, including photos. As presented in Chap. 9: Securement, the prevention of many VAD-associated skin complications is possible with the use of skin barrier films and the correct application of antisepsis, dressing, and securement products. The treatment of skin complications depends upon the diagnosis and severity. Broadhurst et al. (2017) published a consensus and evidence-based algorithm regarding CVAD-associated skin impairment (CASI) relevant to pediatrics. Shown in Fig. 16.2, the CASI algorithm provides direction surrounding the identification and treatment of exit site infection, skin injury (stripping, skin tear), skin irritation/contact dermatitis, and noninfectious weeping.

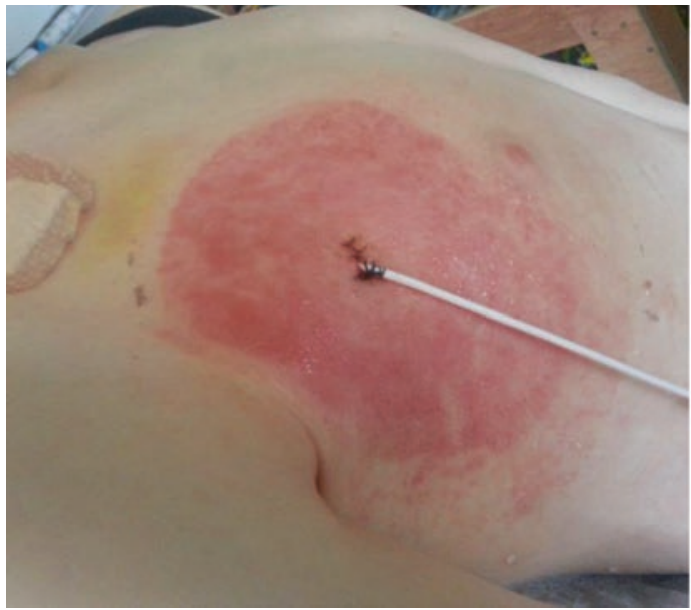

Contact Dermatitis

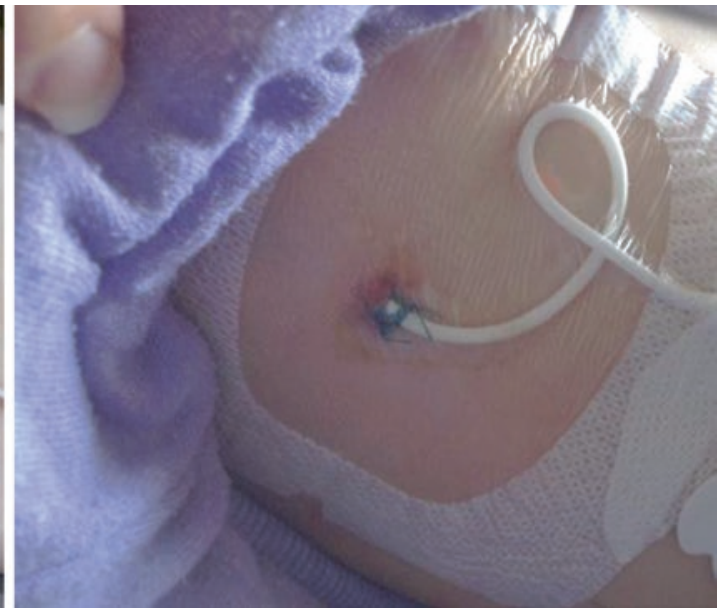

Local Infection

Fig. 16.1 Example of skin complications surrounding pediatric central VADs (used with permission A. Ullman and T. Kleidon) 


\section{CVAD- Associated Skin Impairment (CASI) Algorithm}

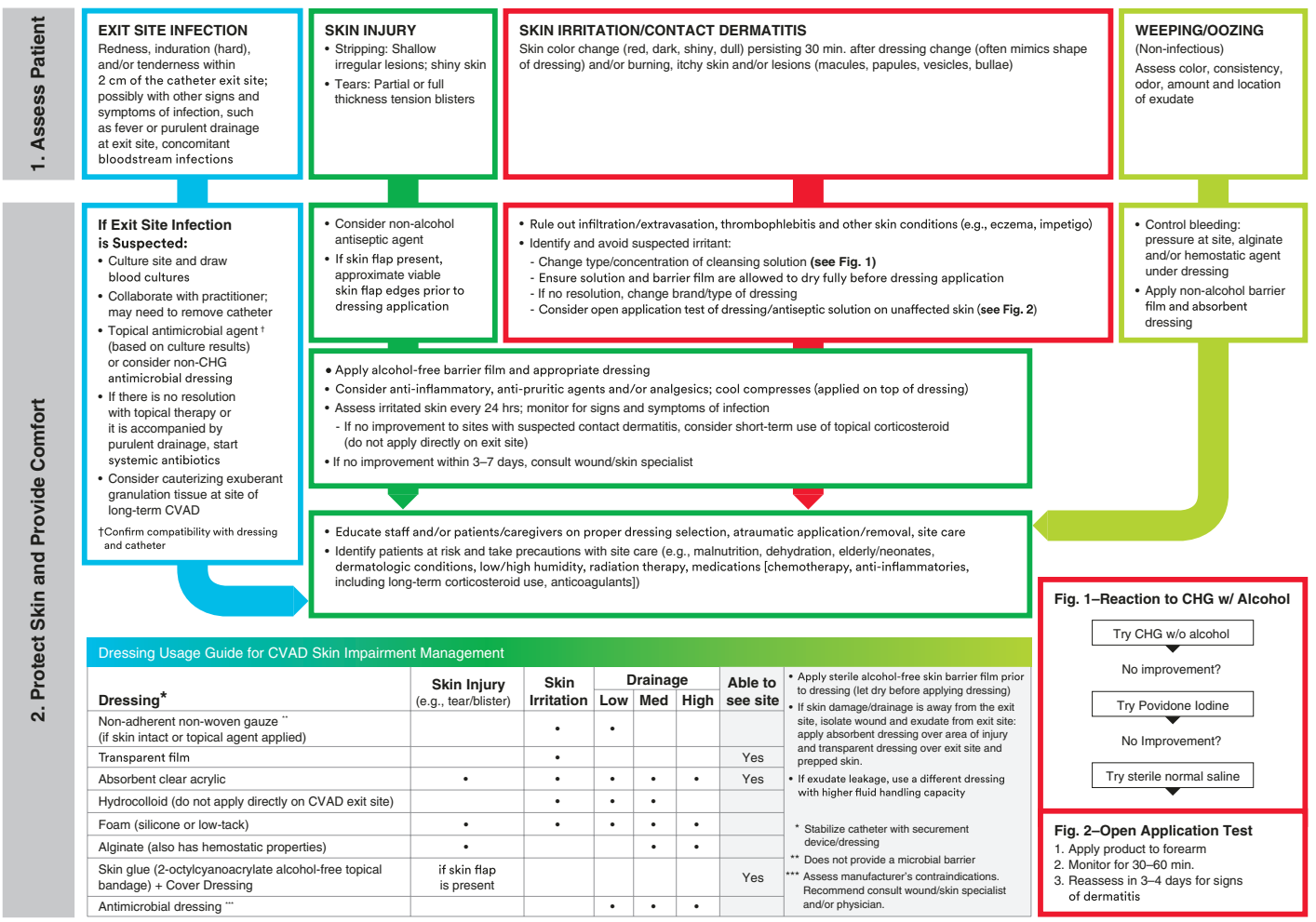

Fig. 16.2 CVAD-associated skin impairment (CASI) algorithm (Broadhurst et al. 2017) (open access, used with permission Broadhurst, Moureau and Ullman)

\subsubsection{Skin Antisepsis}

As described in the previous section, neonatal and pediatric skin have fundamental differences compared to matured skin. Chlorhexidine gluconate $(\mathrm{CHG} ; \geq 0.5 \%)$ in alcohol is demonstrated as being superior to other decontaminants at reducing microbial contamination of VAD insertion sites and thereby preventing VAD-associated infections (Mimoz et al. 2015). However, there are complexities when applying these recommendations to the neonatal and pediatric population. The US Federal Drug Administration (FDA) and clinical guidelines do not recommend, or recommend caution, when using $\mathrm{CHG}$ for infants less than 2 months of age (Federal Drug Administration n.d.; O'Grady et al. 2011). This is due to concerns regarding $\mathrm{CHG}$ absorption and skin irritation. Additionally, skin irritation, including contact dermatitis, has also been frequently reported when using alcohol-based solutions in the neonatal and young infant population. For neonates, young infants and children with impaired skin integrity, the use of povidone iodine, water-based solutions, or other decontaminants should be considered. The risk-to-benefit ratio of using $\mathrm{CHG}$ and alcohol needs to be considered by clinicians. No matter which skin antiseptic agent is used, it is important that the agent is allowed to dry fully prior to dressing application. Wet decontaminants under VAD dressings frequently result in skin irritation and injury.

\subsubsection{Dressing and Securement}

As with the dressing and securement of VADs in other populations, the dressing and securement of pediatric VADs has multiple functions to promote device patency and prevent complication. 
Dressing products cover the VAD wound, providing a protective barrier from contamination from the environment, preventing extra-luminal contamination of the VAD insertion site (Ullman et al. 2015a). Securement products stabilize the VAD, ensuring stability of the device tip location either in a central or peripheral position. Securement products also aim to prevent micromotion of the VAD within the vessel (preventing vessel irritation and thereby thrombosis) and within the wound (preventing wound irritation and thereby local site infection) (Ullman et al. 2015a). The overarching literature regarding the range of products that are available to support VAD security has been presented in Chap. 9: Securement.

Within pediatrics, there are some unique features of VAD dressing and securement that must be considered. Infants and small children are rarely compliant in their dressing and securement application and may intentionally attempt to remove the device. The products need to withstand the mechanics of a crawling infant and a bored, non-compliant toddler. This means additional security and coverage, through splints and devices, are often necessary. Consider anchoring the device in an area that is difficult to reach such as over the infant or toddler shoulder. However, the composite of dressing and securement products in use must facilitate regular assessment of the VAD site to monitor for early signs of infiltration, extravasation, phlebitis, and local site irritation. Dressing and securement products must be easy to apply and remove by a variety of clinicians in challenging circumstances. VAD security and dressing products also need to be appropriately sized, with many of the modern non-suture securement products requiring a large footplate, making them difficult to apply in situations such as a jugular nontunneled central VAD.

Medication-impregnated dressing products involve a slow release of antiseptic solutions onto the VAD insertion site. CHG-impregnated dressing products, either by disk or gel or built into the dressing, have been demonstrated to significantly reduce the rate of central VAD-related bloodstream infections (Ullman et al. 2015b). However, the effectiveness of CHG-impregnated products to prevent infection has not been comprehensively studied in the pediatric population or in devices outside of the critical care setting. While clinical practice guidelines (Loveday et al. 2014; O'Grady et al. 2011) and pragmatism recommend their suitability in most pediatric settings with a high rate of bloodstream infection, caution should again be employed. It is not currently recommended to use CHG-impregnated dressing products in neonates and small infants less than 2 months of age and those with significantly impaired skin integrity surrounding central VAD sites, due to risk of significant skin injuries and irritation. Silver-impregnated dressing products have been successfully used in a single-center neonatal pilot randomized controlled trial without evidence of systemic silver absorption and no evidence of skin damage (Hill et al. 2010). However, the effectiveness of silver-impregnated dressing products for infection prevention has not been demonstrated.

\subsection{Pediatric Vessel Size and Patency}

Typically, the smaller the patient, the smaller the vessels. As detailed in Chap. 14: The Right Device Assessment, for younger children, smaller gauge catheters are used to prevent complications of thrombosis and phlebitis. However, the use of smaller gauge catheters results in higher risk of intraluminal occlusion due to medication precipitate and thrombosis.

\subsection{Flushing}

As detailed in Chap. 19: Flushing, there are a variety of strategies that can be used to promote VAD patency including pulsatile flushing using normal saline and heparin, continuous infusion, and locking. Flushing is commonly used to verify VAD patency and theoretically clear the device between medications that could result in occlusion (Doellman et al. 2015). VADs are generally flushed with normal saline before and after medi- 
cation administration and blood sampling and after an infusion is ceased (Doellman et al. 2015). Routine, pulsatile flushing with normal or heparinized saline is also used between medication administration to create turbulent flow, thereby promoting patency and reducing bacterial catheter colonization. Debate continues regarding the value of heparin flush when a VAD is not in use. Unfortunately, there is limited evidence regarding the optimal flushing frequency and solutions across all populations and even less in pediatrics. The most recent Cochrane systematic review concluded that there was not enough evidence to determine the effects of heparin versus normal saline to prevent occlusion in pediatric CVADs (Bradford et al. 2015), yet it remains the most commonly used flushing and locking solution (Doellman et al. 2015). The additional fluid administered during VAD flushing is an issue of concern for pediatric patients who may be fluid restricted because of their age (e.g., neonates) or medical conditions (e.g., renal disease) (Doellman et al. 2015).

\subsection{Locking}

VAD locking is most commonly used for CVADs and aims to prevent blood reflux during periods of disuse. A variety of heparin doses are used depending upon the device type, length, and the perceived risk of occlusion (see Table 16.1). To prevent occlusion in small-gauge devices (e.g., 1.9Fr PICC), pediatric vascular access specialists may choose to initiate a low-volume continuous infusion of crystalloid solution. While evidence of its effectiveness is poor, it is theorized that the continuous infusion promotes patency by preventing thrombus formation.

Additional lock solutions should be initiated based upon clinical indication. These include fibrinolytic agents, ethanol, antibiotics (e.g., vancomycin), antibiotic-heparin mixes, and taurolidine. There is limited evidence to support the routine prophylactic use of these lock solutions; however, a growing evidence base supports the use of ethanol and taurolidine lock solutions as either prophylaxis or as catheter salvage in pediatric patients at high risk of CVAD-associated bloodstream infection (Liu et al. 2013; Mokha et al. 2017; Raad et al. 2016).

\subsection{Blood Sampling}

Pediatric VADs are frequently used for blood sampling during chronic, acute, and critical illness. While blood sampling from peripheral VAD can be troublesome, and frequently results in device malfunction, blood sampling from CVAD is a common procedure. Despite the ubiquity of blood sampling from pediatric CVADs, there is substantial variation in technique and poor evidence to support good practice. Blood sampling from pediatric VAD is of common practice due to difficulty associated with peripheral phlebotomy, risks associated with iatrogenic anemia, volume depletion,

Table 16.1 Locking guidelines for pediatric CVAD (modified from (Doellman et al. 2015))

\begin{tabular}{|c|c|}
\hline CVAD type and priming volumes & $\begin{array}{l}\text { Locked device (volume, solution, and frequency) } \\
\text { Add volume for add-on devices to priming volume }\end{array}$ \\
\hline $\begin{array}{l}\text { PICCs } \\
\text { Device priming volume ranges from } 0.06 \text { to } 0.6 \mathrm{~mL} \text {. } \\
\text { Check manufacturer guidelines }\end{array}$ & $\begin{array}{l}2 \text { Fr and smaller: continuous infusion preferred or } \\
1 \mathrm{~mL} \text { heparinized saline }(10 \mathrm{U} / \mathrm{mL}) \text { every } 6 \mathrm{~h} \\
2.6 \mathrm{Fr} \text { and larger: } 1-2 \mathrm{~mL} \text { heparinized saline }(10 \mathrm{U} / \\
\mathrm{mL}) \text { every } 12 \mathrm{~h}\end{array}$ \\
\hline $\begin{array}{l}\text { Tunneled and non-tunneled } \\
\text { Device priming volume ranges from } 0.12 \text { to } 1.3 \mathrm{~mL} \text {. } \\
\text { Check manufacturer guidelines }\end{array}$ & 1-3 mL heparinized saline $(10-100 \mathrm{U} / \mathrm{mL})$ every $24 \mathrm{~h}$ \\
\hline $\begin{array}{l}\text { Totally implanted device } \\
\text { Device priming volume ranges from } 0.8 \text { to } 2 \mathrm{~mL} \text { Check } \\
\text { manufacturer guidelines }\end{array}$ & $\begin{array}{l}\text { If used for more than one medication daily: } 3-5 \mathrm{~mL} \\
\text { heparinized saline }(10 \mathrm{U} / \mathrm{mL}) \\
\text { Monthly maintenance flush: } 3-5 \mathrm{~mL} \text { heparinized saline } \\
(100 \mathrm{U} / \mathrm{mL})\end{array}$ \\
\hline
\end{tabular}


catheter occlusion, and CVAD-associated bloodstream infections (Doellman et al. 2015; Ullman et al. 2016).

The three most common techniques for blood sampling from pediatric CVADs are the discard, reinfusion, and push-pull methods. The discard method is common in adult populations and involves the aspiration and then disposal of clearance blood (volume dependent upon line volume), prior to blood sampling. Within pediatrics, this technique can result in significant fluid loss and preventable anemia especially during times of frequent blood sampling (e.g., sepsis). Comparatively, the reinfusion method involves the aspiration of clearance blood sampling and then reinfusion of clearance blood. This technique potentially increases the risk of microbial colonization and thrombosis formation within the clearance volume. The push-pull method involves the multiple rapid infusion and aspiration of first saline, and then blood, through into the syringe, theoretically clearing the line of infusion fluid prior to sampling. This minimizes potential contamination, thrombosis formation, and fluid loss but increases the risk of inaccurate results. Previous clinical trials in pediatrics have demonstrated statistically, but not clinically different results using the push-pull method (Adlard 2008; Barton et al. 2004).

\subsection{Pediatric Vascular Access Skills and Training}

\subsubsection{Clinicians}

As described in Chap. 5: Vascular Access Teams, healthcare institutions must ensure development of vascular access qualified and competent staff, including a central core of vascular access specialists. All clinicians caring for children with VADs must be knowledgeable and skilled in the day-to-day management of the variety of VAD types, the prevention of complications, and postinsertion management strategies. Educational programs must be dynamic and completed by clinicians regularly (not just once). Successful clini- cian educational programs may include a combination of didactic lectures, web-based modules, and high- and low-fidelity simulation, including competency assessment with feedback. Educational programs must incorporate pediatric focused vascular access interventions including the use of child life specialists to promote compliance during procedures such as dressing changes and pain relief. Interdisciplinary clinician training plays a key role in the provision of quality post-insertion VAD care.

Within pediatrics, it is necessary to consider the higher level of advanced skills that are required to manage and problem-solve complex vascular access conditions. Institutions should be supported by a cohort of expert vascular access clinicians who are able to educate and lead highquality post-insertion management of pediatric VADs. These clinicians may also be involved in the leadership of local clinical practice guideline review and implementation.

\subsubsection{Family and Caregivers}

Family and primary caregivers must also be comprehensively educated to manage VADs in the home environment. Home-based healthcare is growing across all specialties, with peripheral and central VADs the cornerstone of many home-based therapies, such as antibiotic and parenteral nutrition administration. These therapies range from short term to lifelong administration. Education and resources need to be provided so that the primary caregivers can manage and problem-solve potentially problematic pediatric VADs.

Family members are a significant resource in the prevention of post-insertion VAD complications in the acute care environment. Primary caregivers are intimately familiar with the complexities of their child and their VAD. Whether a short- or long-term device, they understand their child's reaction to pain, compliance with procedures, and, for those with a long-term vascular access dependency, the distinct tricks that may help promote device patency. Never, never ignore the parents and caregivers. 


\subsection{Conclusion}

The management of pediatric VADs after insertion requires specialist focus. The prevention of post-insertion complications in pediatric VAD must be led by comprehensively trained staff, with easily accessible support from vascular access experts. Evidence-based strategies must be used to prevent complications including infection, occlusion, and site irritation. Parents and caregivers are an important resource to be utilized in the post-insertion phase.

\section{Case Study}

Rosie is a 4-year-old girl, undergoing treatment for relapsed acute myeloid leukemia (AML) including an allogenic bone marrow transplant. Rosie has a tunneled cuffed CVAD that exits through her right chest wall. Rosie developed symptoms of graft versus host disease 2 days ago, with initial symptoms of poor gut absorption. As her vascular access expert, consider:

1. What types of VAD-associated skin complications is Rosie at risk of developing?

2. What products and procedures could be initiated to reduce the risk of Rosie developing a VAD-associated skin complication?

\section{Case Study}

Jacob (18 months) is recovering in pediatric intensive care after cardiac surgery. $\mathrm{He}$ is lightly sedated but highly reliant upon his jugular central VAD for inotropes and other medicines. As his vascular access expert, consider:

1. Would you use a CHG-impregnated dressing product?

2. How would you secure his central VAD considering his risk of removal?

\section{Summary of Key Points}

1. Post-insertion complications are common in pediatrics with $25 \%$ of CVAD and $25-35 \%$ of peripheral VAD failing prior to completion of treatment.

2. Young age and common pediatric conditions impair skin integrity, rendering the promotion of skin health and regular assessment of skin complications surrounding VAD necessary.

3. Skin antisepsis in pediatrics can be challenging. Chlorhexidine use is restricted in infants $<2$ months, and alcohol may also be associated with inflammatory skin conditions. Other decontaminants should be considered, and the risk-tobenefit ratio must be evaluated by clinicians.

4. Young children are at increased risk for accidental and intentional VAD dislodgement. However, products used to promote device security must facilitate regular site assessment and easy application/removal.

5. VAD patency can be challenging, due to small lumen size. Consider the use of continuous infusion to maintain patency for very small lumen devices.

6. Healthcare institutions need to invest in high-quality, dynamic pediatric VAD education, to develop VAD competent interdisciplinary staff.

7. Parents and caregivers are an important resource to prevent VAD complications.

\section{References}

Adlard K. Examining the push-pull method of blood sampling from central venous access devices. J Pediatr Oncol Nurs. 2008;25(4):200-7. https://doi. org/10.1177/1043454208320975.

Barton SJ, Chase T, Latham B, Rayens MK. Comparing two methods to obtain blood specimens from pediatric central venous catheters. J Pediatr Oncol Nurs. 2004;21(6):320-6. https://doi. org/10.1177/1043454204269604. 
Bradford NK, Edwards RM, Chan RJ. Heparin versus $0.9 \%$ sodium chloride intermittent flushing for the prevention of occlusion in long term central venous catheters in infants and children. Cochrane Database Syst Rev. 2015;11:Cd010996. https://doi. org/10.1002/14651858.CD010996.pub2.

Broadhurst D, Moureau N, Ullman AJ. Management of central venous access device-associated skin impairment: an evidence-based algorithm. J Wound Ostomy Continence Nurs. 2017;44(3):211.

Doellman D, Buckner JK, Hudson Garrett J Jr, Catudal JP, Frey AM, Lamagna P, et al. Best practice guidelines in the care and maintenance of pediatric central venous catheters. 2nd ed. Herriman, UT: Association for Vascular Access; 2015.

Federal Drug Administration. Medical devices. https:// www.fda.gov/MedicalDevices/

Hill ML, Baldwin L, Slaughter JC, Walsh WF, Weitkamp JH. A silver-alginate-coated dressing to reduce peripherally inserted central catheter (PICC) infections in NICU patients: a pilot randomized controlled trial. J Perinatol. 2010;30(7):469-73. https://doi. org/10.1038/jp.2009.190.

Liu Y, Zhang AQ, Cao L, Xia HT, Ma JJ. Taurolidine lock solutions for the prevention of catheter-related bloodstream infections: a systematic review and metaanalysis of randomized controlled trials. PLoS One. 2013;8(11):e79417. https://doi.org/10.1371/journal. pone.0079417.

Loveday H, Wilson J, Pratt R, Golsorkhi M, Tingle A, Bak A, et al. EPIC3: national evidence-based guidelines for preventing healthcare-associated infections in NHS hospitals in England. J Hosp Infect. 2014;86(Suppl 1):S1-70. https://doi.org/10.1016/ s0195-6701(13)60012-2.

Malyon L, Ullman AJ, Phillips N, Young J, Kleidon T, Murfield J, Rickard CM. Peripheral intravenous catheter duration and failure in paediatric acute care: a prospective cohort study. Emerg Med Australas. 2014;26(6):602-8. https://doi. org/10.1111/1742-6723.12305.

Mimoz O, Lucet JC, Kerforne T, Pascal J, Souweine B, Goudet V, et al. Skin antisepsis with chlorhexidinealcohol versus povidone iodine-alcohol, with and without skin scrubbing, for prevention of intravascular-catheter-related infection (CLEAN): an open-label, multicentre, randomised, controlled, twoby-two factorial trial. Lancet. 2015;386(10008):206977. https://doi.org/10.1016/s0140-6736(15)00244-5.

Mokha JS, Davidovics ZH, Samela K, Emerick K. Effects of ethanol lock therapy on central line infections and mechanical problems in children with intestinal failure. JPEN J Parenter Enteral Nutr. 2017;41(4):625-31. https://doi.org/10.1177/0148607115625057.

O'Grady NP, Alexander M, Burns LA, Dellinger EP, Garland J, O'Heard S, et al. Guidelines for the prevention of intravascular catheter-related infections. Clin Infect Dis. 2011;52(9):e162-93.

Raad I, Chaftari AM, Zakhour R, Jordan M, Al Hamal $\mathrm{Z}$, Jiang Y, et al. Successful salvage of central venous catheters in patients with catheter-related or central line-associated bloodstream infections by using a catheter lock solution consisting of minocycline, EDTA, and 25\% ethanol. Antimicrob Agents Chemother. 2016;60(6):3426-32. https://doi.org/10.1128/ aac.02565-15.

Rozsa AP, Bell AJ, Tiitinen MT, Richards S, Newall F. Peripheral intravenous catheters in a paediatric population: circumstances of removal and time in situ. Neonatal Paediatr Child Health Nurs. 2015;18(3):18-24.

Ullman AJ, Cooke M, Rickard C. Examining the role of securement and dressing products to prevent central venous access device failure: a narrative review. J Assoc Vasc Access. 2015a;20(2):99-110.

Ullman AJ, Cooke ML, Mitchell M, Lin F, New K, Long DA, et al. Dressings and securement devices for central venous catheters (CVC). Cochrane Database Syst Rev. 2015b;9:Cd010367. https://doi. org/10.1002/14651858.CD010367.pub2.

Ullman AJ, Marsh N, Mihala G, Cooke M, Rickard CM. Complications of central venous access devices: a systematic review. Pediatrics. 2015c;136(5):e1331-44. https://doi.org/10.1542/peds.2015-1507.

Ullman AJ, Keogh S, Coyer F, Long DA, New K, Rickard CM. 'True Blood' the critical care story: an audit of blood sampling practice across three adult, paediatric and neonatal intensive care settings. Aust Crit Care. 2016;29(2):90-5. https://doi.org/10.1016/j. aucc.2015.06.002.

Open Access This chapter is licensed under the terms of the Creative Commons Attribution 4.0 International License (http://creativecommons.org/licenses/by/4.0/), which permits use, sharing, adaptation, distribution and reproduction in any medium or format, as long as you give appropriate credit to the original author(s) and the source, provide a link to the Creative Commons license and indicate if changes were made.

The images or other third party material in this chapter are included in the chapter's Creative Commons license, unless indicated otherwise in a credit line to the material. If material is not included in the chapter's Creative Commons license and your intended use is not permitted by statutory regulation or exceeds the permitted use, you will need to obtain permission directly from the copyright holder.

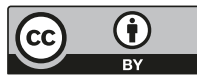

